\title{
SUMOylation of HP1a supports association with ncRNA to define responsiveness of breast cancer cells to chemotherapy
}

\author{
Feng-Ming Lin ${ }^{1}$, Santosh Kumar², Jing Ren ${ }^{2}$, Samaneh Karami², Shaymaa Bahnassy ${ }^{2}$, \\ Yue $\mathrm{Li}^{3}$, Xiaofeng Zheng ${ }^{4}$, Jing Wang ${ }^{4}$ and Tasneem Bawa-Khalfe ${ }^{2}$ \\ 1 Department of Cardiology, The University of Texas MD Anderson Cancer Center, Houston, TX, USA \\ ${ }^{2}$ Center for Nuclear Receptors and Cell Signaling, Department of Biology and Biochemistry, University of Houston, Houston, \\ TX, USA \\ ${ }^{3}$ Department of Integrative Biology and Pharmacology, University of Texas Health Science Center at Houston, Houston, TX, \\ USA \\ ${ }^{4}$ Department of Bioinformatics and Computational Biology, The University of Texas MD Anderson Cancer Center, Houston, \\ TX, USA \\ Correspondence to: Tasneem Bawa-Khalfe, email: tbawa-khalfe@uh.edu
}

Keywords: SUMO, SENP7, HPla, nCRNA, Rad51C

Received: March 31, $2016 \quad$ Accepted: April 03, $2016 \quad$ Published: April 14, 2016

\section{ABSTRACT}

Epigenetic reprogramming allows cancer cells to bypass normal checkpoints and potentiate aberrant proliferation. Several chromatin regulators are subject to reversible SUMO-modification but little is known about how SUMOylation of chromatinremodelers modulates the cancer epigenome. Recently, we demonstrated that SUMOprotease SENP7L is upregulated in aggressive BCa and maintains hypoSUMOylated heterochromatin protein 1-a (HP1a). Canonical models define HP1a as a "reader" of repressive $\mathrm{H} 3 \mathrm{~K} 9 \mathrm{~m} 3$ marks that supports constitutive heterochromatin. It is unclear how SUMOylation affects HP1a function in BCa cells. This report shows HP1a SUMOdynamics are closely regulated in a complex with SENP7L and SUMO-E3 Polycomb-2 (PC2/CBX4). This complex accumulates at $\mathrm{H} 3 \mathrm{~K} 9 \mathrm{~m} 3$ sites, hypoSUMOylates HP1a and PC2, and reduces PC2's SUMO-E3 activity. HyperSUMO conditions cause complex dissociation, SUMOylation of PC2 and HP1a, and recruitment of SUMOylated HP1a to multiple DNA-repair genes including Rad51C. SUMOylated HP1a's enrichment at euchromatin requires chromatin-bound non-coding RNA (ncRNA), reduces Rad51C protein, and increases DNA-breaks in BCa cells. Hence, HP1a SUMOylation and consistently low SENP7L increase efficacy of DNA-damaging chemotherapeutic agents. BCa patients on chemotherapy that express low SENP7L exhibit greater survival rates than patients with high SENP7L. Collectively, these studies suggest that SUMOylated HP1a is a critical epigenetic-regulator of DNA-repair in BCa that could define chemotherapy responsiveness.

\section{INTRODUCTION}

SUMO post-translational modification (PTM) of protein substrates is important for normal cell physiology but is thrown off kilter with the onset of cancer $[1,2]$. This process or SUMOylation requires one or more of 3 SUMO isoforms, E1 activating molecules, and a sole E2 conjugating enzyme Ubc9. Often SUMO E3 ligases facilitate interaction between $\mathrm{Ubc} 9$ and the target protein and thereby potentiate the conjugation reaction. In contrast, SENPs reverse this PTM or deSUMOylate via hydrolysis of the isopeptide bond between SUMO and the target $[3,4]$. The two arms of SUMO-PTM work in concert to dictate the level of SUMOylated cellular substrates.

Several components of the SUMO machinery are localized at select chromatin loci. We and others have demonstrated that full-length SUMO protease SENP7L 
is present at the tri-methylated histone 3 lysine 9 residue (H3K9m3, [5-7]). SUMO E3 ligase Polycomb-2 (PC2/ $\mathrm{CBX} 4)$ can associate with $\mathrm{H} 3 \mathrm{~K} 9 \mathrm{~m} 3$ and an additional repressive mark tri-methylated histone 3 lysine 27 residue (H3K27m3) as established in recombinant in vitro systems [8]. In vivo studies indicate that recruitment of PC2 to $\mathrm{H} 3 \mathrm{~K} 27 \mathrm{~m} 3$ is potentiated with SUMO-PTM of PC2 [9]; in contrast, little is known about what regulates localization of PC2 to H3K9m3. Enrichment of SENP7L to H3K9m3 requires interaction with the canonical $\mathrm{H} 3 \mathrm{~K} 9 \mathrm{~m} 3$ reader heterochromatin protein-1 alpha (HP1 $\alpha$ ).

Previous studies established the critical role of HP1 $\alpha$ in stabilizing constitutive heterochromatic regions, specifically the pericentromere and subtelomere $[10,11]$. Recent studies provide increasing evidence to support the recruitment and function of $\mathrm{HP} 1 \alpha$ at transcriptional active euchromatin sites $[5,12,13]$. Unlike HP1 $\alpha$ 's recruitment to heterochromatin, little is known about what directs HP $1 \alpha$ to euchromatin sites. While heterochromatin is $\mathrm{H} 3 \mathrm{~K} 9 \mathrm{~m} 3$-rich, $\mathrm{HP} 1 \alpha$ is present at the pericentromeric region prior to the establishment of $\mathrm{H} 3 \mathrm{~K} 9 \mathrm{~m} 3$. H3K9m3independent recruitment of HP1 $\alpha$ to the pericentromere occurs predominantly via HP1 $\alpha$ 's association with cisacting non-coding RNA (ncRNA) of the $\alpha$-satellite repeats $[7,10,14]$. It is unknown whether chromatin-bound ncRNA could direct HP1 $\alpha$ to euchromatin sites.

Recently we reported that HP1 $\alpha$ 's enrichment to select euchromatin loci dictates the aggressiveness of breast cancer (BCa) cells [5]. In BCa cells, SUMO-PTM of HP1 $\alpha$ facilitates HP1 $\alpha$ 's localization to the promoter of proliferation- and mesenchymal-inducing genes and subsequently silences transcription of these genes. Immunofluorescence data shows persistent binding of SUMOylated HP1 $\alpha$ outside constitutive heterochromatin. However, chromatin-binding profiles of HP1 $\alpha$ are lacking. This would provide greater understanding of $\mathrm{HP} 1 \alpha$ 's function in $\mathrm{BCa}$ especially as we and others have demonstrated HP1 $\alpha$ levels correlate with metastatic disease [5, 15-17]. In highly invasive BCa cells, HP1 $\alpha$ is hypoSUMOylated but it is unclear whether reduction of HP1 $\alpha$ SUMOylation affects transcription of additional BCa cell survival genes..

In the present study, we define how and why SUMOylation of HP1 $\alpha$ is closely guarded in BCa cells. HP1 $\alpha$ concurrently binds the SUMO E3 ligase PC2 and isopeptidase SENP7L; this complex maintains hypoSUMOylated HP1 $\alpha$ levels in BCa cells. Knockdown of SENP7L or PC2 overexpression increases HP1 $\alpha$ SUMOylation. HyperSUMOylated HP1 $\alpha$ exhibits an altered chromatin-binding profile as compared to its un-modified counterpart with elevated binding to 5'UTR and promoter regions of multiple genes including DNA-damage response (DDR) genes. Recruitment of the SUMOylated HP1 $\alpha$ to these euchromatic sites requires chromatin-bound ncRNA in $\mathrm{BCa}$ cells. HP1 $\alpha$ hyperSUMOyation and SENP7L depletion increase the sensitivity of $\mathrm{BCa}$ cells to DNA-damaging chemotherapeutics. Consistently BCa patients with lower SENP7L exhibit a better response to chemotherapy.

\section{RESULTS}

\section{PC2 serves as SUMO E3 ligase and interacting partner for HP1a}

Recently, we reported that HP1 $\alpha$ is more readily SUMOylated in non-cancerous versus cancer mammary epithelial cells [5]. However, it is unknown what components of the SUMO-conjugating machinery facilitate HP1 $\alpha$ SUMOylation. Since PC2 also interacts with $\mathrm{H} 3 \mathrm{~K} 9 \mathrm{~m} 3$ sites, we postulated that overexpression of this SUMO E3 ligase would potentiate SUMO-PTM of HP1 $\alpha$. Indeed, isolated endogenous chromatin-bound $\mathrm{HP} 1 \alpha$ is readily SUMOylated with induction of PC2 in MCF7 cells (bracket highlights SUMOylated HP1 $\alpha$, Figure 1A). While PC2 substantially enhances HP1 $\alpha$ SUMOylation, co-transfection of HP1 $\alpha$ with other chromatin-associated SUMO E3 ligases, specifically protein inhibitor of activated STAT 4 (PIAS4), LIM domain only 2 (LMO2), and RAN binding protein 2 (RanBP2, [9, 18-20]) only modestly increases poly-SUMO modification of HP1 $\alpha$ (Figure S1A).

Interestingly, $\mathrm{PC} 2$ directly interacts with its substrate HP1 $\alpha$ in chromatin fractions (Figure 1A). The amino acid sequence of PC2 includes two well-defined SUMO-interacting motifs (SIM) as well as a lysine residue near the C-terminus that serves as a SUMO-acceptor site $[21,22]$. Previous studies established that PC2 requires both SIMs for SUMO E3 ligase activity and PC2 autoSUMOylation [21, 22]. PC2 also includes a potential HP1 $\alpha$-interaction motif (HIM) or PxVxL sequence that exists as part of the second SIM (SIM2, Figure 1B). Hence we evaluated how critical the converging amino acids of the HIM/SIM2 sequence are for PC2's interaction with HP1 $\alpha$. Wild-type forms of HP1 $\alpha$ and PC2 bind efficiently (lane 1, Figure 1C). While mutation of IVIVSIM1 sequence (SIM1m) reduces interaction with HP1 $\alpha$ wt (lane 2, Figure 1C), replacement of the VILL sequence (SIM2m) ablates the ability of PC2 to bind HP1 $\alpha$-wt (lane 3 , Figure 1C). Hence, SIM2 overlaps with a canonical HIM, which supports PC2 binding to HP1 $\alpha$.

Compared to SIM2m, SIM1m promotes less in vitro SUMOylation of HP1 $\alpha$ (lane 2 versus lane 3, Figure 1D) and SUMO3 poly-chain formation (lane 5 versus lane 6, Figure 1D). This suggests SIM1, more than SIM2, contributes to PC2's E3 ligase function. However, both PC2-SIMs are required for optimum enzymatic activity (lane 1 and lane 4, Figure 1D). We next investigated whether association with HP1 $\alpha$ affects PC2's ligase function. In a cell-free system, increasing amounts of 
recombinant HP1 $\alpha$ protein reduce the ability of wt-PC2 to initiate poly-SUMO3 chain formation (lane 3-5, Figure 1E). Partial loss of HIM (SIM2m) restores SUMO E3 activity even with the addition of increasing recombinant
HP1 $\alpha$ (lane 6-8, Figure 1E). Inversely, inhibition of SUMO ligase activity regulates PC2's interaction with $\mathrm{HP} 1 \alpha$. PC2 expresses a serine-SIM consensus sequence [21]; on an additional SUMO ligase PIAS1, casein kinase 2 (CK2)
A.

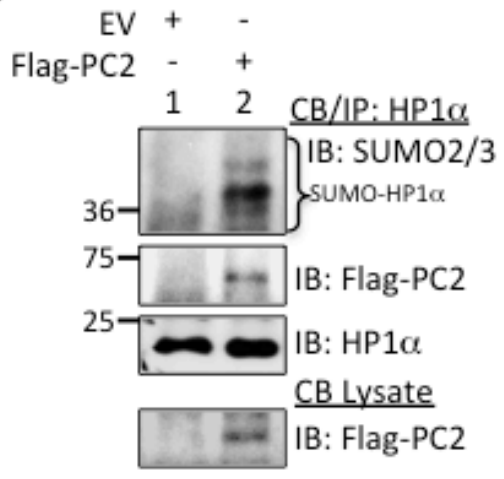

B.

\begin{tabular}{|c|c|c|}
\hline & IM1 & HIM/SIM2 SUMC \\
\hline WT & |IVIV| & PeVILL VKPE \\
\hline 110 & $258-261$ & $69 \quad 491.494$ \\
\hline & & M2 SUM \\
\hline CD & AAAA & PeVILL VKPE \\
\hline & SIN & \\
\hline 100 & IVIV & PeAA \\
\hline
\end{tabular}

c.

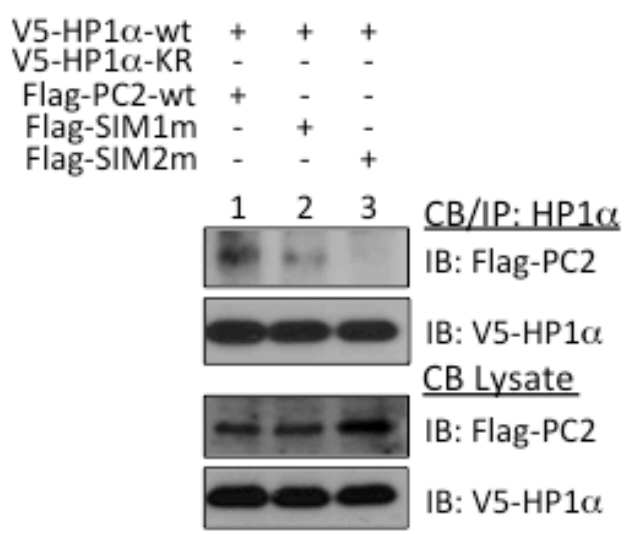

D.

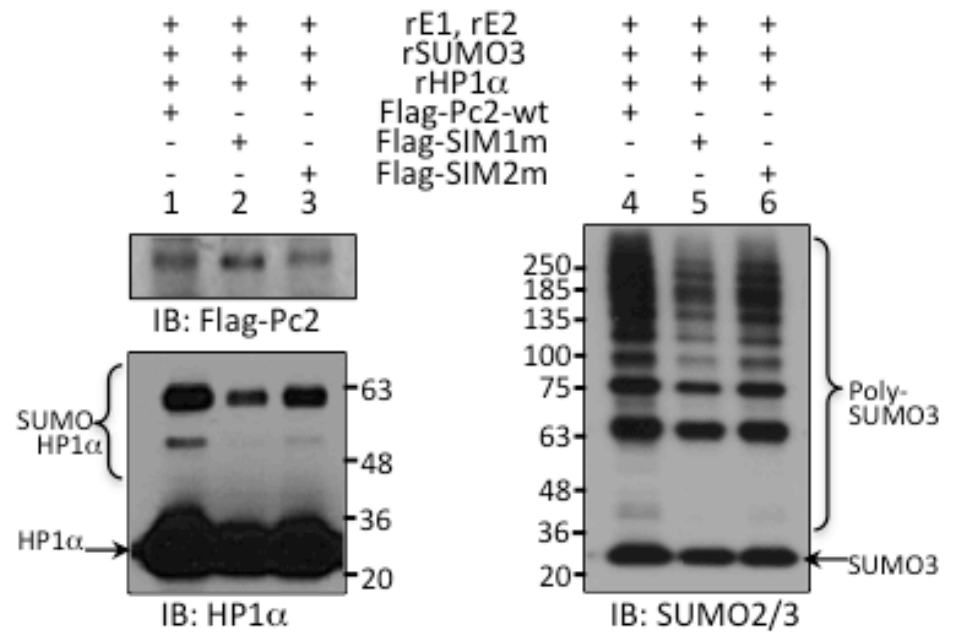

E.

$\begin{array}{rcccccccc}\text { rE1, rE2 } & + & + & + & + & + & + & + & + \\ \text { rSUMO3m } & + & - & - & - & - & - & - & - \\ \text { rSUMO3 } & - & + & + & + & + & + & + & + \\ \text { rHP1 } \alpha & - & - & & & & & & \\ \text { Flag-PC2-wt } & - & - & + & + & + & - & - & - \\ \text { Flag-SIM2m } & - & - & - & - & - & + & + & + \\ & 1 & 2 & 3 & 4 & 5 & 6 & 7 & 8\end{array}$

IB: SUMO3

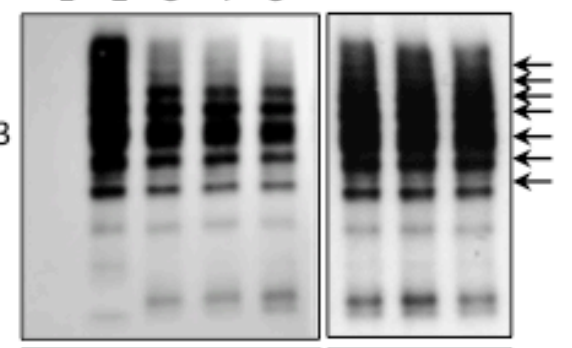

IB: Flag-Pc2

IB: HP1 $1 \alpha$

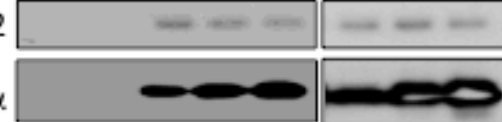

Figure 1: HP1 $\alpha$ interacts with PC2 to regulate PC's SUMO E3 ligase activity. A. Chromatin-bound endogenous HP1 $\alpha$ associates with and is a target for PC2. Endogenous HP1 $\alpha$ was immunoprecipitated from MCF7 cells expressing empty vector (EV) or flag-tagged PC2. Immunoblots using SUMO2/3 and Flag antibodies confirmed HP1 $\alpha$ modification and protein-protein interaction. B. Schematic representation of PC2's 2 SUMO-interaction motifs (SIM), HP1 $\alpha$-interaction motif (HIM), and single SUMO-conjugation site in relation to the chromodomain (CD). The hydrophobic amino acids were mutated to alanine to generate the appropriate SIM1 and SIM2 mutants. C. HP1 $\alpha$-interaction motif is required for PC2-HP1 $\alpha$ binding. MCF7 cells were incubated with either wild-type or PC2 plasmids with mutations to either 1 of the 2 SIMs. Association of PC2 with HP1 $\alpha$ was assessed via SDS-PAGE. D. PC2-SIM1 affects in vitro HP1 $\alpha$ SUMOylation. Purified Flag-tagged wild-type or SIM-mutant PC2 was incubated with recombinant SUMO machinery and HP1 $\alpha$ and subsequently subject to immunoblotting. Poly-SUMO chains (brackets labeling SUMO-HP1 $\alpha$ and Poly-SUMO3) and unmodified HP1 $\alpha$ and SUMO3 protein (arrows) is indicated. E. The E3 activity of wt-PC2, but not SIM2 m, is reduced with increasing concentrations of HP1 $\alpha$. An in vitro SUMOylation assay was used to evaluate poly-SUMO chains in the presence of increasing recombinant HP1 $\alpha$. Arrows SUMO3 bands in PC2-wt samples affected by the addition of recombinant $\mathrm{HP} 1 \alpha(\mathrm{rHP} 1 \alpha)$. 
phosphorylates the serine residue adjacent to a SIM to potentiate ligase activity [23]. Treatment with a CK2 inhibitor reduces PC2 phosphorylation (Supplemental Figure S1B) and SUMOylation (Supplemental Figure $\mathrm{S} 1 \mathrm{C})$. This reduction of PC2 auto-SUMOylation and corresponding E3-activity concurrently increases PC2HP $1 \alpha$ interaction (Supplemental Figure S1C).

\section{HP1 $\alpha$ interacts with SUMO E3-ligase PC2 and Isopeptidase SENP7L simultaneously}

HP1 $\alpha$ interacts with SENP7L through multiple HIMs [5-7]. Hence we questioned whether HP1 $\alpha$ associates with PC2 and SENP7L simultaneously or independently. Chromatin-bound HP1 $\alpha$ efficiently interacts with PC2 following induction of SENP7L in MCF7 cells (lane 4, Figure 2A). A SENP7 isoform, which lacks one HIM sequence (SENP7S), neither binds HP1 $\alpha$ (lane 3, Figure 2A and [5]) nor supports PC2HP1 $\alpha$ interaction (lane 3, Figure 2A). While increasing SUMO3 supports poly-SUMO conjugation of PC2, concurrent SENP7L induction prompts deSUMOylation of chromatin-bound PC2 (lane 2 and 3, respectively, Figure 2B). Inversely, siRNA-targeted knockdown of SENP7 (siSENP7) enhances PC2 SUMOylation in MCF7 cells (Figure 2C). The same SENP7 knockdown conditions also increase HP1 $\alpha$ SUMOylation and concomitant PC2 overexpression further potentiates the SUMOylated HP1 $\alpha$ population (Supplemental Figure S2A and S2B). Hence, both PC2 and HP1 $\alpha$ are substrates for the isopeptidase
SENP7L. Interestingly, the loss of SENP7 also reduces PC2's interaction with HP1 $\alpha$ (Figure 2C), suggesting that SENP7-mediated deSUMOylation is important for PC2HP1 $\alpha$ binding.

Since both PC2 (Figure 1C) and SENP7L express HIMs, it is likely that HP1 $\alpha$ serves as a scaffold for the recruitment of both SUMO components to the $\mathrm{H} 3 \mathrm{~K} 9 \mathrm{~m} 3$ mark. Both PC2 and SENP7L localize to the nucleus predominantly (yellow arrows, Figure 3A). Treatment of MCF7 cells with siRNA that efficiently reduces HP1 $\alpha$ protein levels ( the subcellular distribution of SENP7L but not PC2. Specifically, HP1 $\alpha$ loss increases SENP7L localization to the cytosol (white arrows, Figure 3A); BCa cells expressing SENP7L in the nucleus are reduced while cells with even or greater distribution of SENP7L in the cytosol are significantly increased $(p<0.01$, Student's $t$-test, Figure $3 \mathrm{~B}$ and Supplemental Figure S3B). This observation correlates with previous studies that $\mathrm{HP} 1 \alpha$ is required for localization of SENP7 in the nucleus and more specifically at the constitutive pericentric heterochromatin $[7,24]$. Although siHP1 $\alpha$ treatment did not alter subcellular distribution of PC2 (Figure 3A), HP1 $\alpha$ loss decreases the enrichment of PC2 at H3K9m3 marks (Figure 3C). Inversely, PC2's localization at H3K27m3 sites (Figure 3C) and SUMOylation (Supplemental Figure $\mathrm{S} 3 \mathrm{C}$ ) is enhanced with HP1 $\alpha$ knockdown. Similarly, hyperSUMO conditions reduce $\mathrm{PC} 2$ 's recruitment to $\mathrm{H} 3 \mathrm{~K} 9 \mathrm{~m} 3$ sites (Figure 3D). PC2-HP1 $\alpha$ interaction also diminishes with SUMO3 induction (Figure 3C); analogously PC2-HP1 $\alpha$ binding decreases with enhanced
A.

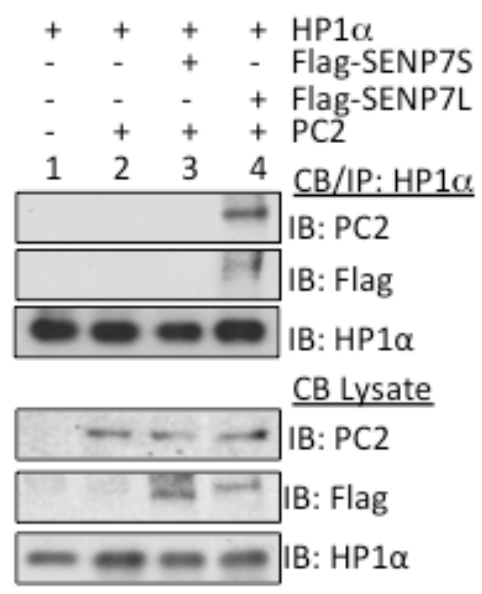

B.

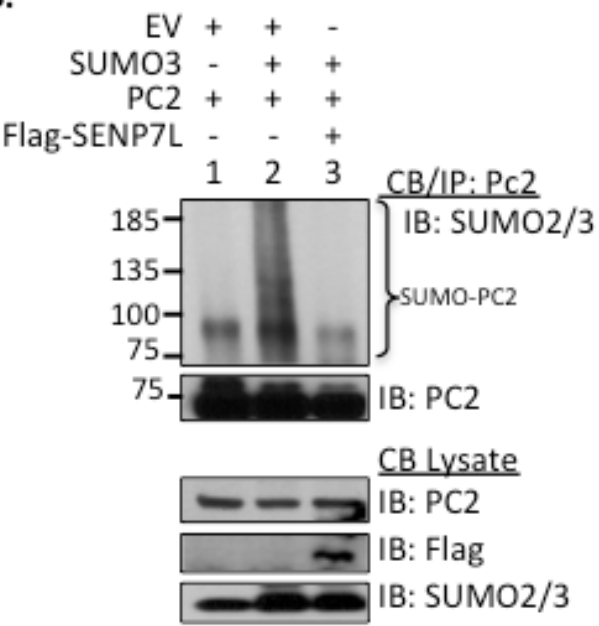

C.

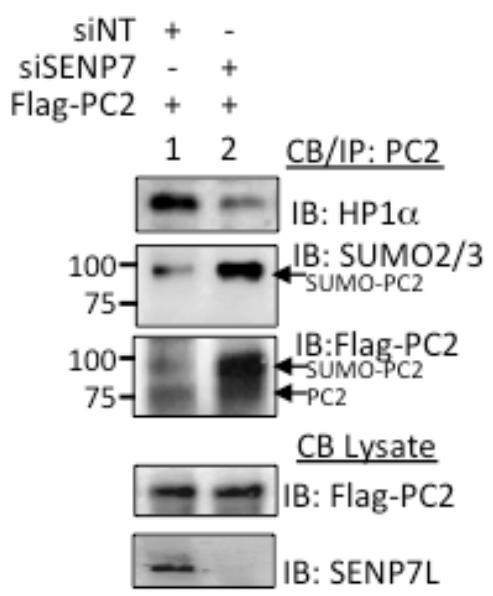

Figure 2: SENP7L regulates PC2-HP1 $\alpha$ interaction and PC2 SUMOylation. A. PC2, HP1 $\alpha$, and SENP7L interact at the chromatin. Chromatin-bound HP1 $\alpha$ was isolated from MCF7 cells and interaction with PC2 and/or select SENP7 isoforms was evaluated with Western Blot analysis. B. SENP7L deSUMOylates PC2. In in vivo SUMOylation studies, modification of isolated chromatin-bound PC2 is identified with SUMO2/3 antibody in the presence and absence of SENP7L. C. SENP7L dictates PC2's SUMOylation and binding to HP1 $\alpha$. MCF7 cells were treated with the appropriate siRNA for $48 \mathrm{hr}$ and subsequently the modification state of the chromatin-bound PC2 was established as described above. 
SUMOylation of PC2 (Figure 2C). Hence, it is likely that un-modified PC2 associates predominantly with HP1 $\alpha$ at the $\mathrm{H} 3 \mathrm{~K} 9 \mathrm{~m} 3$ mark.

\section{SUMOylated HP1a is recruited to ncRNA- richchromatin loci}

A previous report suggests that interaction with mouse SENP7 docks HP1 $\alpha$ at H3K9m3-rich pericentromeric heterochromatin [24]. Interestingly, hyperSUMOylated HP1 $\alpha$ does not efficiently bind either SENP7 or PC2 (Supplemental Figure S3D). Hence, we postulated that SUMOylated HP1 $\alpha$ could exhibit a different chromatin binding profile than its unmodified counterpart. To test this, ChIP-Seq experiments were utilized to evaluate the distribution of wild-type HP1 $\alpha$ (wt-HP1 $\alpha$ ) versus a SUMOylated HP1 $\alpha$ mimetic (S-HP1) that includes HA-tagged SUMO3 close to the endogenous SUMO-acceptor site of HP1 $\alpha$ [5]. A high number of sequencing reads from the samples aligned to the hg19 reference genome (Supplemental Table S1). Biostatistical validation was performed to filter peaks based on high confidence (FDR 0.05) and no overlap with input peaks (Supplemental Table S2). Of the 632 and 1012 filtered peaks for wt-HP1 and S-HP1 $\alpha$ respectively, the two constructs share only 257 binding sites (Figure 4A). The $\mathrm{S}-\mathrm{HP} 1 \alpha$ has a greater number of "unique" binding sites or regions that do not overlap with wt-HP1 $\alpha$ (Figure 4A). These unique $\mathrm{S}-\mathrm{HP} 1 \alpha$ binding sites are detected at genecoding and non-coding loci; specifically, S-HP1 binds the 5'UTRs, promoters, and introns in intragenic regions as

A.
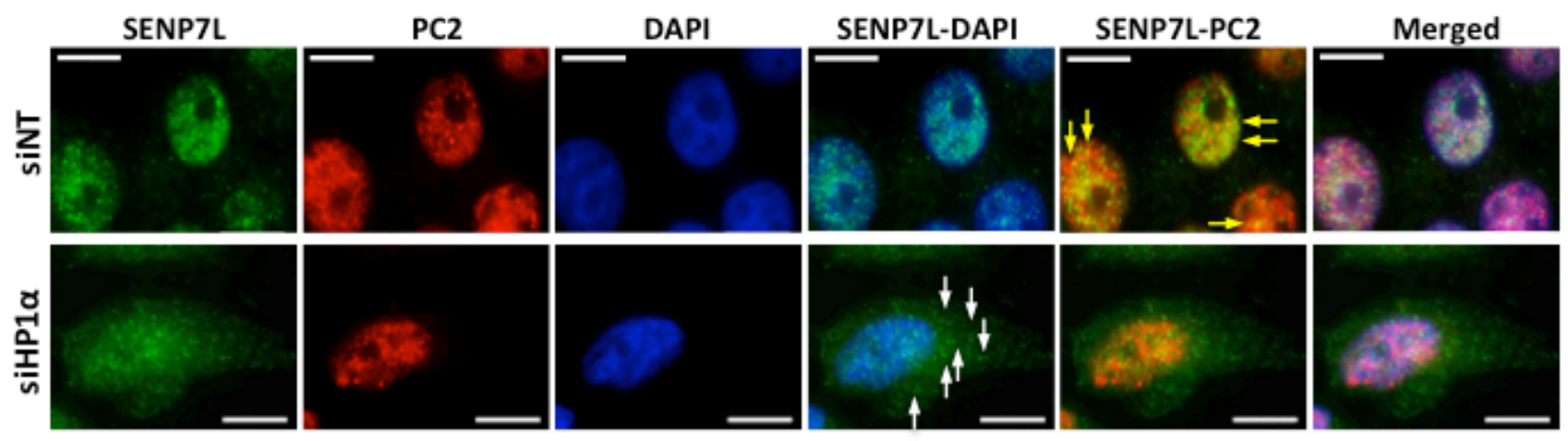

B.

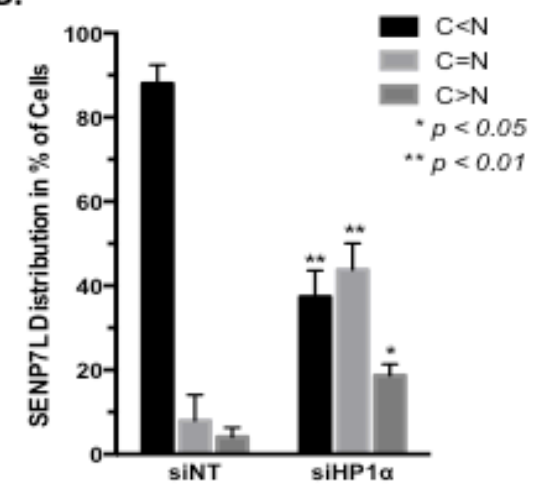

C.

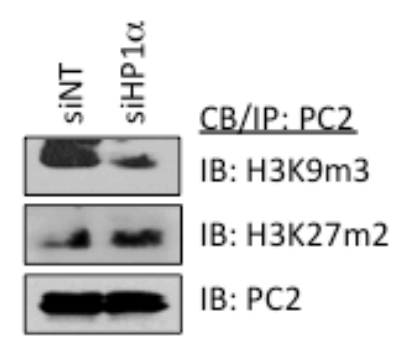

D.

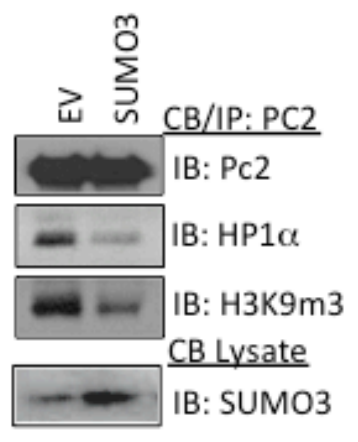

Figure 3: HP1 $\alpha$ directs the localization of PC2 and SENP7L. A.-B. HP1 $\alpha$ loss alters SENP7L subcellular localization. Cells were incubated with either non-targeting (siNT) or HP1 $\alpha$-specific ( $\operatorname{siHP} 1 \alpha$ ) siRNA for $48 \mathrm{hr}$. Immunofluorescence was performed with SENP7L and PC2 antibodies and nuclei stained with DAPI. Total cells and SENP7L distribution was evaluated in randomly selected 20x magnification fields (siNT: $n=3$ and siHP1 $\alpha: n=7$ ); percent of cells with cytosolic (C) versus nuclear (N) SENP7L were analyzed using ANOVA and Tukey's post-hoc. C. PC2's enrichment at H3K9m3 requires HP1 $\alpha$. Chromatin-bound PC2 was isolated from MCF7 cells treated with either siNT or siHP1 as described above. Western blot analysis was performed to evaluate PC2's association with modified histones tails, specifically H3K9m3 and H3K27m3. D. HyperSUMO conditions reduce PC2's association with both H3K9m3 and HP1 $\alpha$. MCF7 cells were exposed to elevated SUMO3 levels for $24 \mathrm{hr}$ and binding partners of endogenous PC2 was evaluated using the immunoprecipitation/ immunoblot techniques. 
well as promoters of long ncRNA (lncRNA) and noncoding regions for both lncRNA and microRNA (miRNA) (Figure 4B). Many of the novel loci for S-HP1 $\alpha$ include genes that contribute to the DDR, specifically Rad51C, PPM1D, PPM1E, BRIP1. Alignment of the binding profiles of wt-versus S-HP1 $\alpha$ on the Rad51C gene loci exhibit interesting similarities and differences (Figure 4C). Both wt- and S-HP1 bind at the Rad51C promoter and 5'-UTR (gray box, Figure 4C) but a greater detection of $\mathrm{S}-\mathrm{HP} 1 \alpha$ is observed adjacent to the promoter and in intron 7 (purple box, Figure 4C).

S-HP1 $\alpha$ appears readily bound to genes like $\operatorname{Rad} 51 C$ that express unspliced lncRNA (Figure 4B). A previous report suggests that SUMOylated HP1 $\alpha$ interacts more readily with ncRNA at the constitutive heterochromatin in mouse fibroblasts [14]. Hence we first evaluated the binding profile of wt- and S-HP1 $\alpha$ with published transbinding regions for 2 lncRNAs TERC and HOTAIR [25]. Significantly more S-HP1 $\alpha$ (33 sites), but not wt-HP1 $\alpha$ (4 sites), binding sites either directly overlapped with or were within close proximity (200bp) to either TERC or HOTAIR trans-binding regions. To further test whether ncRNA facilitate SUMOylated HP1 $\alpha$ 's recruitment to active chromatin sites, a modified ChIP protocol was developed. The RNA-DNA complex was disrupted with RNase $\mathrm{H}$ treatment prior to ChIP with the HP1 $\alpha$ antibody; the treatment did not cause adverse RNA and/or DNA degradation in the harvested cell fractions. The recruitment of S-HP1 $\alpha$ to the pericentromeric $\alpha$-satellite repeats was significantly attenuated with RNase $\mathrm{H}$ treatment (Figure 5A). This is consistent with previous immunofluorescence studies in which hyperSUMO conditions promote
A.

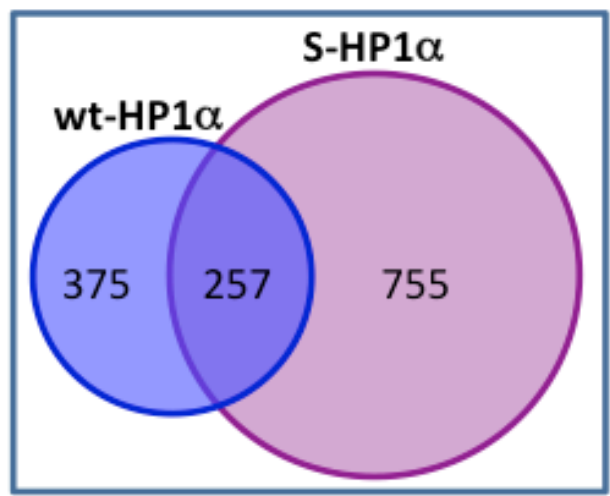

B.

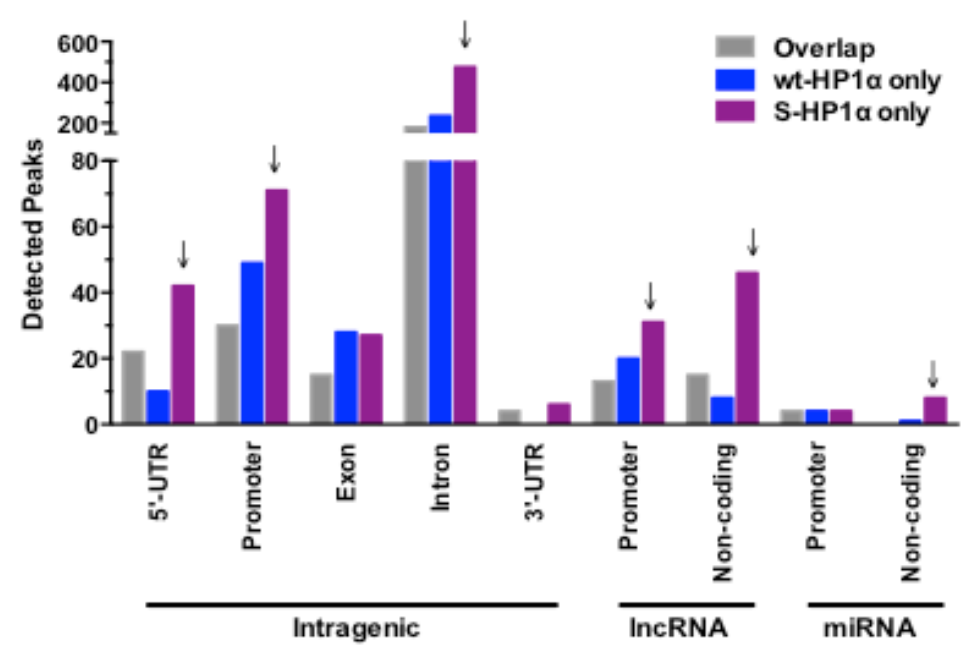

C.

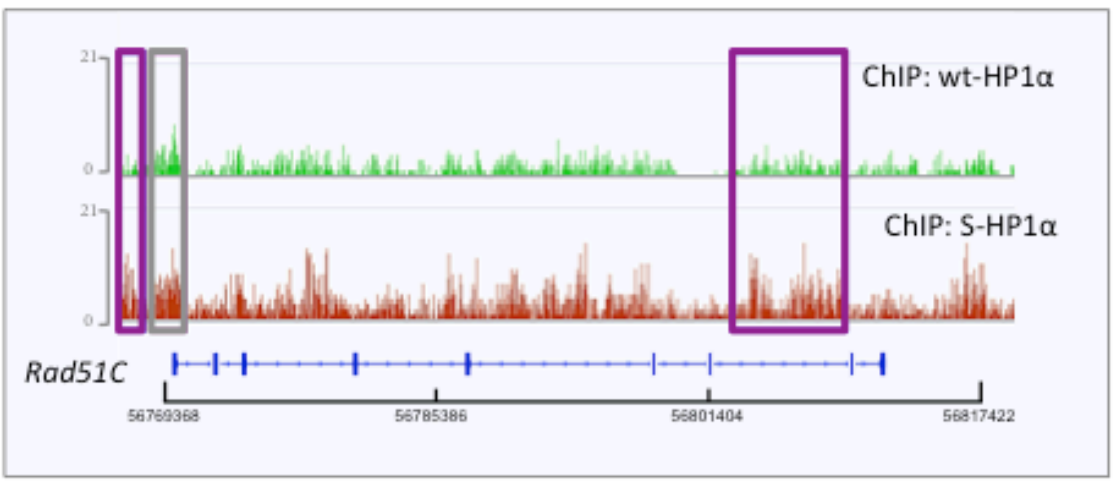

Figure 4: HP1 $\alpha$ SUMOylation alters its chromatin binding profile. A. Venn diagram of the total binding loci of wild-type and SUMO3-fused HP1 $\alpha$ (wt- and S-HP1 $\alpha$ ) as identified by ChIP-Seq analysis. B. S-HP1 $\alpha$ binds more 5'-UTR, promoters, and introns in intragenic regions and regions transcribing ncRNA. Graph represents distribution of detected peaks (binding sites) of wt and S-HP1 $\alpha$ at indicated intragenic region. Gray bars indicate binding sites shared by both HP1 $\alpha$ types (Overlap) while binding sites unique to wt-HP1 $\alpha$ or S-HP1 $\alpha$ only are labeled appropriately. Arrows highlight chromosomal regions with a difference between binding profiles of S-HP1 $\alpha$ and wt-HP1 $\alpha$. C. ChIP-Seq track at the Rad51C loci that highlights the number and position of the binding sites for wt- and S-HP1 $\alpha$. Purple box indicates a unique S-HP1 $\alpha$ binding site while gray box is common to both wt and S-HP1 $\alpha$. 
the $\alpha$-satellite-dependent recruitment of HP1 $\alpha$ to the pericentromere [14]. In addition, the enrichment of $\mathrm{S}-\mathrm{HP} 1 \alpha$ at the heterochromatic telomere is reduced with RNase $\mathrm{H}$ treatment (Figure 5A). An intact RNA-DNA complex is also required for the localization of S-HP1 $\alpha$ to several, but not all, ChIP-Seq-identified euchromatic sites (Figure 5A). Specifically RNase treatment only affects SHP1 $\alpha$ enrichment at the E2F-responsive gene DHFR but not TS or $c$-Myc ( $p<0.01$, Student's $t$-test, Figure 5A). Similarly, loss of the RNA-DNA complex affects S-HP1 $\alpha$
A.

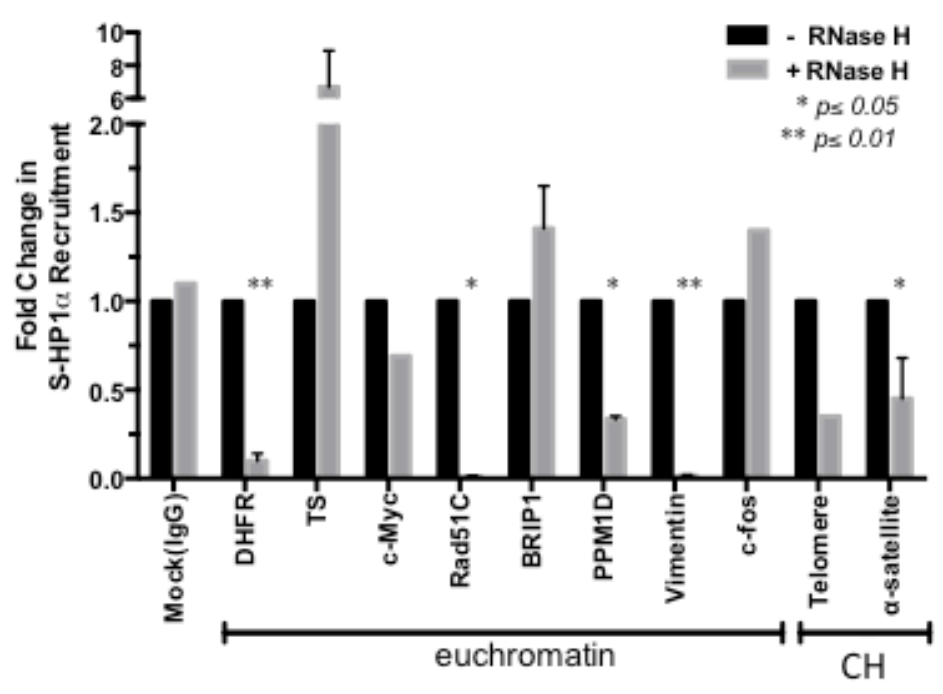

B.

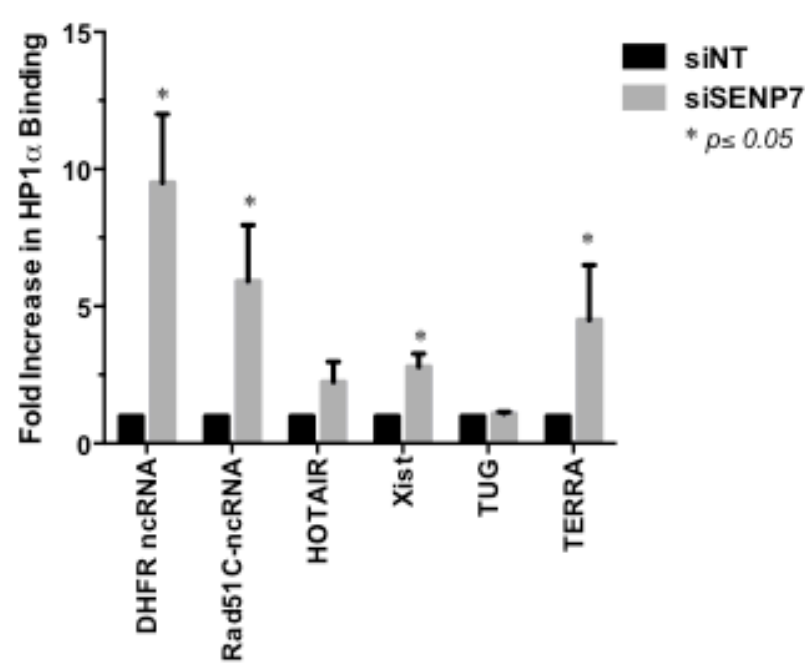

C.

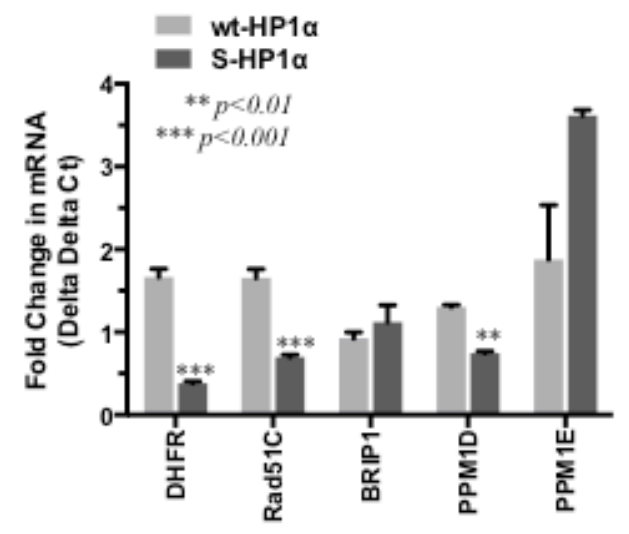

D.

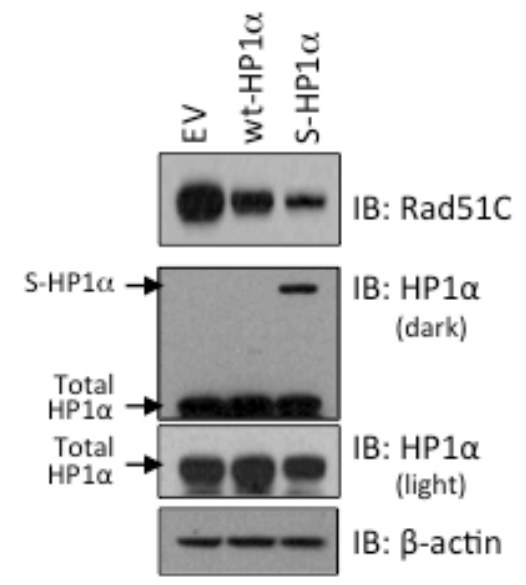

Figure 5: ncRNA-mediated recruitment of SUMOylated HP1 $\alpha$ to DDR-regulating gene loci. A. RNA/DNA complex required for the recruitment of HP1 $\alpha$ to select chromatin loci. MCF7 cells were treated with RNase H to disrupt RNA-DNA interaction prior to ChIP of the S-HP1 $\alpha$ protein. Real-time PCR was performed to identify S-HP1 $\alpha$ recruitment to specific chromatin loci; $\mathrm{CH}$ represents constitutive heterochromatin sites. Student's $t$-test on raw $\mathrm{C}_{\mathrm{T}}$ data was used to evaluate for statistical significance with $p$-values $<0.05$ indicated with an asterisk. B. HP1 $\alpha$ associates with lncRNA in the absence of SENP7L. Following either non-targeting or SENP7-specific siRNA treatment for $48 \mathrm{hr}$, chromatin-bound RNA that associates with HP1 $\alpha$ was isolated via chromatin-RNA immunoprecipitation assay (Ch-RIP). Primers for select ncRNA were used to detect the immunoprecipitated RNA from each treatment group. Bar graph indicates the mean $\pm \mathrm{SEM}$ of three independent Ch-RIP experiments. C. SUMOylated HP1 $\alpha$ reduces select DDR gene transcripts. An empty vector, wild-type HP1 $\alpha$ (wt-HP1 $\alpha$ ), or SUMO3-fused HP1 $\alpha$ (S-HP1 $\alpha$ ) plasmid was overexpressed in MCF7 cells and RNA was isolated. Subsequently real-time PCR analysis was conducted with the appropriate primers. The graph represents mRNA levels of either wt-HP1 $\alpha$ or S-HP1 $\alpha$ treated cells normalized to levels in cells with empty vector; Student's $t$-test indicated significance. D. SUMOylated HP1 $\alpha$ reduces Rad51C protein. Cells expressing either empty vector (EV), wild-type HP1 $\alpha$, or S-HP1 $\alpha$ were lysed and subject to SDS-PAGE to evaluate Rad51C levels. Total HP1 $\alpha$ represents both endogenous and exogenous HP1 $\alpha$. Blots are representative of 2 independent experiments. 
at the Rad51C and PPM1D gene loci but not BRIP1 ( $p<$ 0.05 , Student's $t$-test, Figure 5A). Interestingly, S-HP1 $\alpha$ is lost at chromatin loci that exhibit known and predicted ncRNA; specifically DHFR, Rad51C, PPMID, Vimentin, telomere, and $\alpha$-satellite (Figure 5A, [26-28]).

To evaluate the relationship between HP1 $\alpha$ and ncRNA further, chromatin-associated RNA was isolated with a chromatin-RIP (Ch-RIP). Prior to Ch-
A.
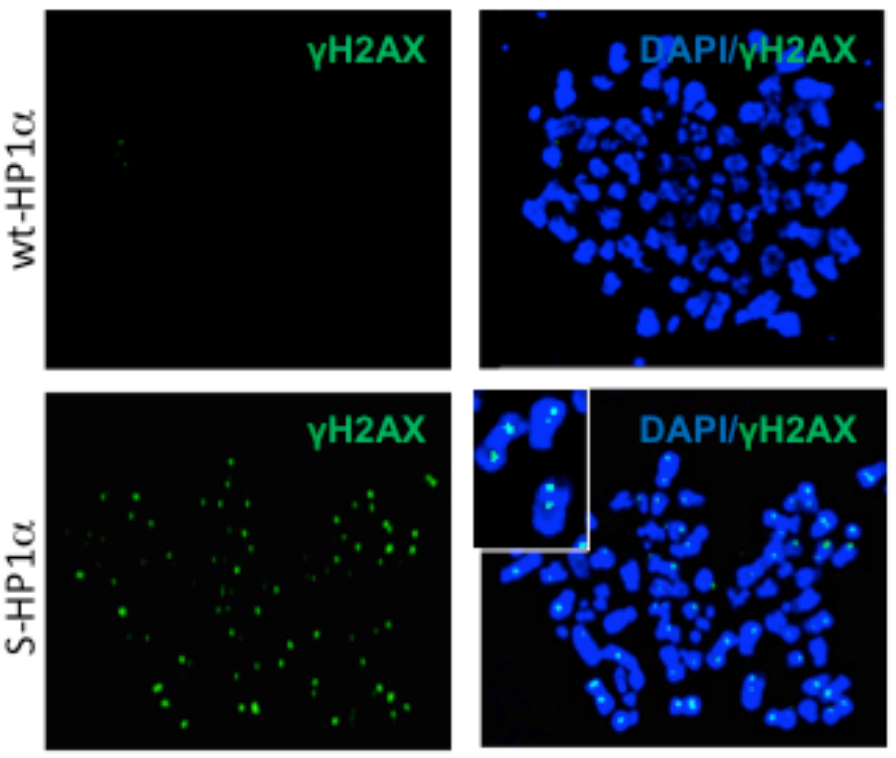

C.
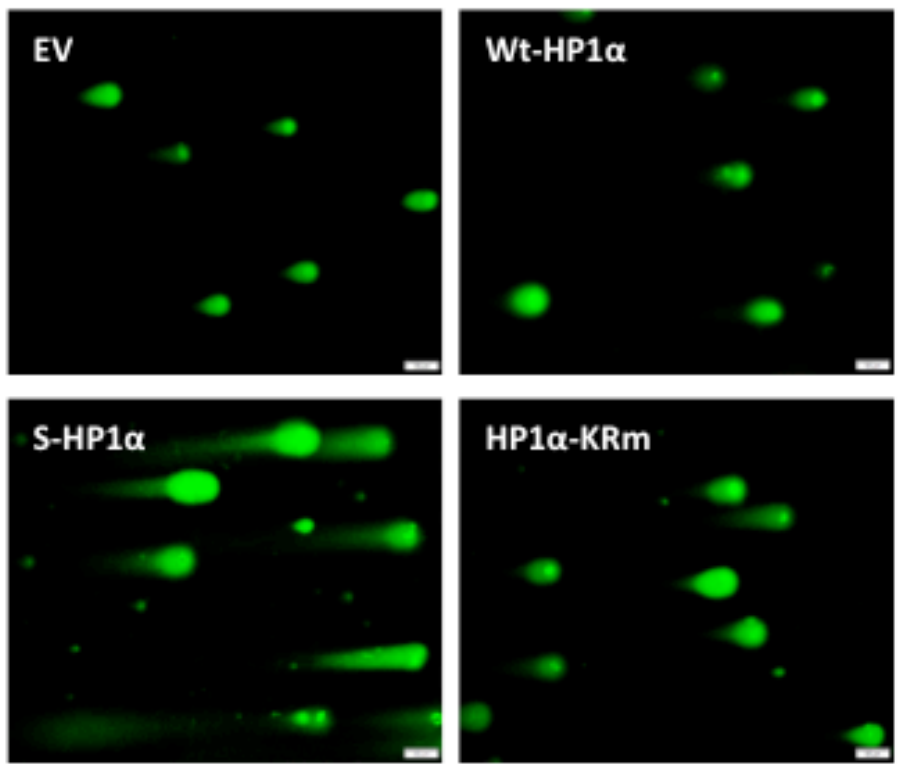

B.

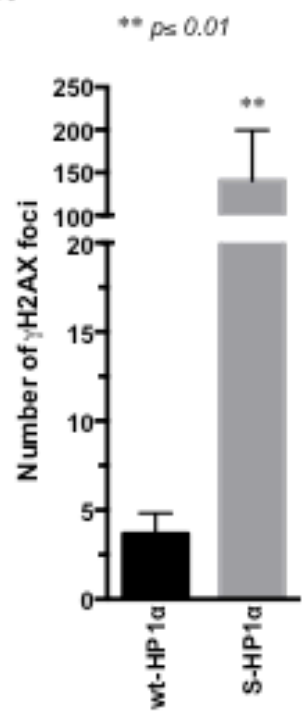

D.

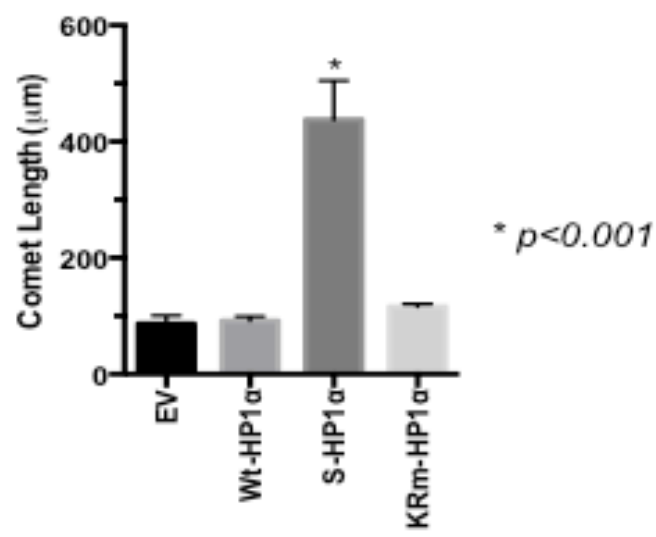

Figure 6: SUMOylation of HP1 $\alpha$ promotes DNA-damage. A.-B. DNA breaks persist with induction of S-HP1 $\alpha$. Immunofluorescence with $\gamma$-H2AX antibody indicated DNA-damage on chromosome-spread MCF7 samples. For each of the two treatment groups, three 100x magnification fields were selected at random and $\gamma$-H2AX foci present on the chromatin were counted. Student's $t$-test was used to evaluate for statistical significance; asterisk presents p-value. C.-D. SUMO-deficient HP1 $\alpha$ does not induce DNA breaks. MCF7 cells were transfected with empty vector (EV), wild-type HP1 $\alpha$ (wt-HP1 $\alpha$ ), SUMO3-fused HP1 $\alpha$ (S-HP1 $1 \alpha$ ) or HP $1 \alpha-K R m$ plasmid and then harvested for evaluation of DNA breaks using the comet assay. A gray scale bar at the bottom right of each image represents $50 \mu \mathrm{m}$. D. The length of the comet was determined using the bar scale as a reference. Statistical significance is indicated as defined using ANOVA and Tukey's post-hoc tests. 
A.

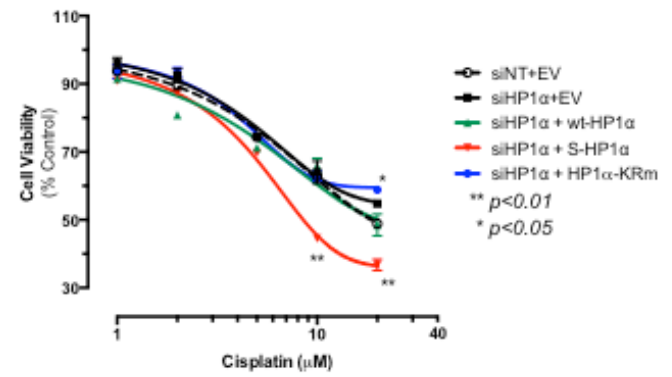

C.

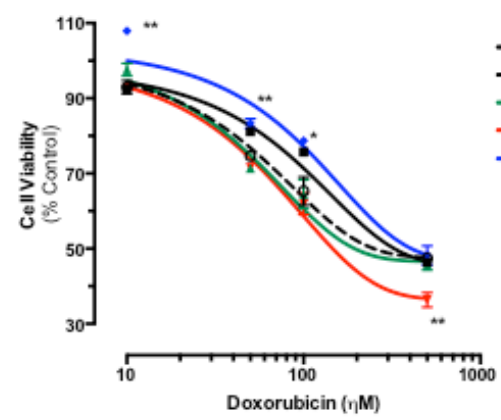

E.

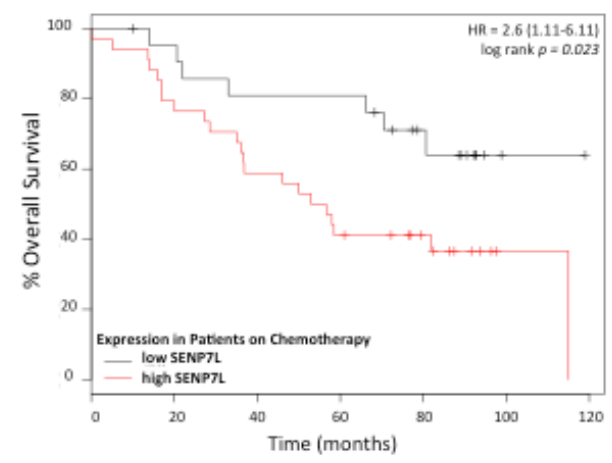

B.

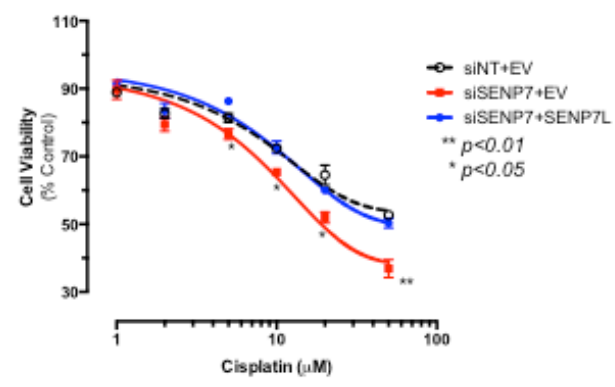

D.

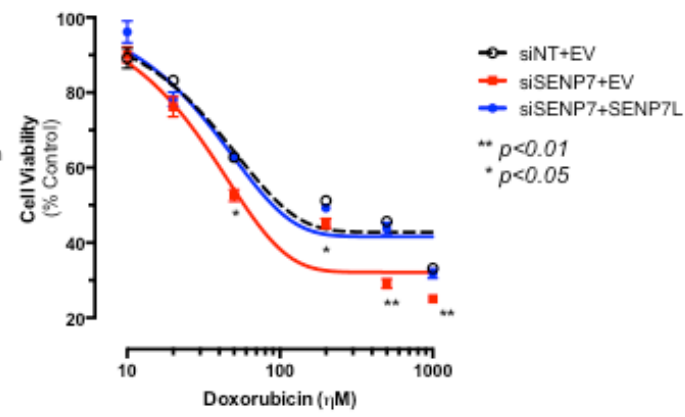

F.

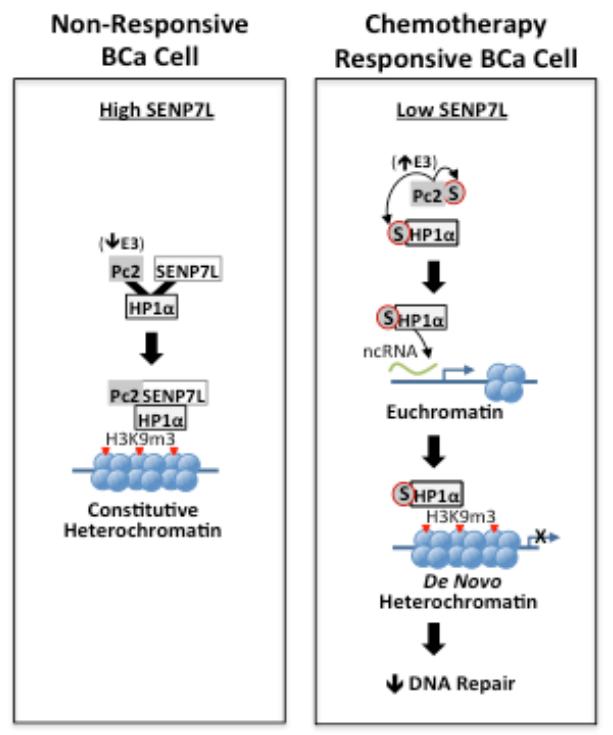

Figure 7: HP1 $\alpha$ SUMOylation status and SENP7L expression predict sensitivity of BCa cells to chemotherapy. A. HP1 $\alpha$ SUMOylation affects cisplatin efficacy. Endogenous HP1 $\alpha$ was knockdown in MCF7 cells with siRNA (siHP1 $\alpha$ ) and reconstituted with wt-HP1 $\alpha$, S-HP1 $\alpha$, and HP1 $\alpha-\mathrm{KRm}$. Cell viability was assessed after 48h incubation with various concentrations of cisplatin. Asterisks represent statistical significance as compared to siNT+EV controls and derived by ANOVA and Dunnett's post-hoc test. B. Loss of SENP7L increases cisplatin efficacy. Cells were treated with either siNT or SENP7-targeting siRNA (siSENP7) concurrently with either empty vector (EV) or SENP7L prior to exposure to increasing concentrations of cisplatin. Asterisks indicate a significant difference as compared to control siNT+EV treated cells; p-values derived following ANOVA and Dunnett's post-hoc test. C.-D. Analogous transfection conditions and statistical analysis as described above was utilized to evaluate the affects of $48 \mathrm{~h}$ doxorubicin treatment on MCF7 cell viability. S-HP1 $\alpha$ reduces while HP1 $\alpha-\mathrm{KRm}$ increases the number of viable cells following doxorubicin treatment C.. Knockdown of SENP7 reduces cell number while reconstitution with SENP7L rescues this effect D.. E. Low SENP7L correlates with greater survival for BCa patients on chemotherapy. Kaplan-Meier plot of chemotherapy-treated BCa patients separated into low and high SENP7L gene expression levels using the KMPLOT database. HR represents the hazard ratio at 95\% confidence and the p-value of $p=0.023$ indicates statistical significance. $\mathbf{F}$. Schematic highlighting the data presented in this manuscript and explained in detail in the main text. 
RIP, hyperSUMOylation of HP1 $\alpha$ was achieved in MCF7 cells with siRNA-targeted knockdown of HP1 $\alpha$ -interacting partner SENP7L (Supplemental Figure S2A and previously [5]. As others have established the ability of HP1 $\alpha$ to interact with ncRNA at the pericentromeric and subtelomeric region $[14,29,30]$, HP1 $\alpha$ 's association with the telomeric TERRA ncRNA served as control. HyperSUMOylation of HP1 $\alpha$, with SENP7L reduction, enhances the ability of HP1 $\alpha$ to bind cis-acting ncRNA TERRA and Xist (Figure 5B). A more modest, but not statistically significant, interaction between HP1 $\alpha$ and trans-acting HOTAIR is observed while no change in TUG ncRNA is detected with SENP7 knockdown (Figure 5B). SENP7-siRNA treatment significantly increases HP1 $\alpha$ 's association with two additional ncRNA, promoterassociated DHFR-ncRNA and the ncRNA transcript of the Rad51C gene NR_103873 (Figure 5B). While the DHFR-ncRNA is known to mediate $D H F R$ transcription $[26,27]$, the function of Rad51C-ncRNA is unknown. Our data suggests that the Rad51C ncRNA can be isolated in chromatin-bound RNA fractions and interacts with HP1 $\alpha$ (Figure 5B).

The recruitment of HP $1 \alpha$ to the Rad51C promoter serves to regulate expression of Rad51C mRNA (Figure $5 \mathrm{C}$ and primers Supplemental Table S5). Induction of wtHP1 $\alpha$ consistently also reduces Rad51C protein levels as compared to empty vector control (lane 2 versus lane 1, Figure 5D). SUMO PTM of HP1 $\alpha$ potentiates this response as the most dramatic loss of $\operatorname{Rad} 51 \mathrm{C}$ is observed with overexpression of S-HP1 $\alpha$ in MCF7 cells (lane 3 versus lanes 1 and 2, respectively, Figure 5D).

\section{HP1 $\alpha$ SUMOylation promotes DNA-damage and correlates with chemotherapy sensitivity}

Since the Rad51 family is a major mediator of the DDR, we postulated that S-HP1 $\alpha$ would impair the DDR and support the accumulation of double-strand breaks (DSB) in BCa cells. MCF7 cells were assessed for $\gamma-\mathrm{H} 2 \mathrm{AX}$ foci, a mark for DSB. Chromatin from cells with wt-HP1 $\alpha$ did not exhibit incorporation of the phosphorylated $\mathrm{H} 2 \mathrm{AX}$ variant (Figure 6A). In contrast, transfection of the S-HP1 $\alpha$ significantly increases $\gamma-\mathrm{H} 2 \mathrm{AX}$ foci on chromatin (Figure 6A and 6B). Similar results were obtained with a comet assay; specifically, elevation of S-HP1 $\alpha$, but not wt-HP1 $\alpha$, causes DNA breaks (Figure $6 \mathrm{C}$ and 6D). In contrast, overexpression of an HP1 $\alpha$ mutant that is SUMO deficient (HP1 $\alpha-\mathrm{KRm},[5])$ does not damage DNA (Figure 6C and 6D). Hence, the data supports that S-HP1 $\alpha$ reduces the ability of the cell to resolve the damaged DNA.

Rad51C and DDR regulate effectiveness of DNAdamaging chemotherapeutic agents like cisplatin [31]. Hence, we next evaluated whether the SUMOylation status of HP1 $\alpha$ dictates sensitivity of $\mathrm{BCa}$ cells to cisplatin treatment. Cisplatin concentration-response assessment of MCF7 cells was performed following knockdown of endogenous HP $1 \alpha$ and reconstitution of cells with wild-type, SUMO-fused, or SUMO-deficient HP1 $\alpha$ (wt-HP1 $\alpha$, S-HP1 $\alpha$, and HP1 $\alpha-K R m$, respectively Supplemental Figure S4A and Figure 7A). Induction of S-HP1 $\alpha$ significantly increased while HP1 $\alpha-\mathrm{KRm}$ decreased cisplatin efficacy (ANOVA, Dunnett's posthoc test, $p<0.01$ and $p<0.05$ respectively, Figure 7A). Consistently, loss of SENP7L, which potentiates HP1 $\alpha$ SUMOylation (Supplemental Figure S2A), also enhances the response of $\mathrm{BCa}$ cells to cisplatin treatment $(p<0.01$, ANOVA and Dunnett's test, Figure 7B). Treatment with an anthracycline-based chemotherapeutic doxorubicin produces similar results with S-HP1 $\alpha$ and SENP7L knockdown increasing doxorubicin efficacy (ANOVA, Dunnett's test for efficacy $p<0.01$, Figure 7C and 7D). Using two publically available data-sets (GSE1456 and GSE3494, $n=56$ ), we next evaluated the correlation between SENP7L expression and overall survival of patients on systemic chemotherapy treatment. SENP7L levels inversely correlate with overall survival for these patients; individuals on chemotherapeutics with low SENP7L mRNA exhibit significantly longer survival time than those patients with high SENP7L mRNA levels ( $p=0.023$, Figure 4F). Interestingly, these patients on chemotherapy with low SENP7L also have a greater probability of relapse-free survival ( $p$ $=0.126$, Supplemental Figure S4B). Hence, it is likely that low SENP7L levels would indicate good response to chemotherapy.

\section{DISCUSSION}

In vitro studies report the high binding affinity of $\mathrm{PC} 2$ for $\mathrm{H} 3 \mathrm{~K} 9 \mathrm{~m} 3$ over $\mathrm{H} 3 \mathrm{~K} 27 \mathrm{~m} 3$ peptides [8]. However, in vivo studies have focused primarily on $\mathrm{PC} 2$ 's recruitment to and function at $\mathrm{H} 3 \mathrm{~K} 27 \mathrm{~m} 3$ sites. We now demonstrate that PC2 is localized at endogenous $\mathrm{H} 3 \mathrm{~K} 9 \mathrm{~m} 3$ sites in $\mathrm{BCa}$ cells (Figure 3C and Figure 3D). Collectively the data suggests that enrichment of PC2 to $\mathrm{H} 3 \mathrm{~K} 9 \mathrm{~m} 3$ is dependent on 1) interaction with $\mathrm{HP} 1 \alpha$ and 2) the SUMOylation status of PC2. First, PC2 exhibits a canonical HP1 $\alpha$ binding motif that is required for PC2-HP1 $\alpha$ association (Figure $1 \mathrm{~B}$ and 1C). Second, HP1 $\alpha$ preferentially associates with hypo-SUMOylated PC2 (Figure 2C and Supplemental Figure S1C). Third, HP1 $\alpha$ loss reduces $\mathrm{PC} 2-\mathrm{H} 3 \mathrm{~K} 9 \mathrm{~m} 3$ association (Figure 3C). Fourth, SUMO3 induction enhances PC2 SUMOylation (lane 2, Figure 2B) but reduce PC2's interaction with both $\mathrm{HP} 1 \alpha$ and $\mathrm{H} 3 \mathrm{~K} 9 \mathrm{~m} 3$. In BCa cells, SUMOylated PC2 is recruited to $\mathrm{H} 3 \mathrm{~K} 27 \mathrm{~m} 3$ (Figure 3C and Supplemental Figure S3C); similarly a previous study reports SUMOylation of PC2 increases H3K27m3 binding in mouse embryonic fibroblasts [9]. Hence while SUMOylated PC2 is enriched at $\mathrm{H} 3 \mathrm{~K} 27 \mathrm{~m} 3$, un-modified 
PC2 associates with $\mathrm{HP} 1 \alpha$ at $\mathrm{H} 3 \mathrm{~K} 9 \mathrm{~m} 3$ sites.

SUMOylated PC2 at $\mathrm{H} 3 \mathrm{~K} 27 \mathrm{~m} 3$ functions to recruit additional PRC1 complex members, specifically Ring1b and Bmi1, to repress transcription at specific gene loci [9]. In contrast, $\mathrm{H} 3 \mathrm{~K} 9 \mathrm{~m} 3$ enrichment serves as a "repressive hub" to sequester un-modified PC2 and provides an alternative regulatory control of PC2's SUMO E3 ligase activity. Optimum PC2 SUMO ligase function requires two SIMs ([21] and Figure 1D). Unlike SIM1, SIM2 overlaps with a HP1 $\alpha$-binding site. HP1 $\alpha$-binding reduces PC2's SUMO ligase activity as demonstrated in Figure 1D and 1E. Exactly how PC2-SIMs regulate ligase function is unclear; it is proposed that the SIMs could either modulate association with SUMO-loaded Ubc9 or orient SUMO for transfer from Ubc9 to substrate [21]. HP1 $\alpha$ could masks PC2-SIM2 and through steric hindrance, perturbs its full SUMO ligase activity. Alternatively, binding to HP1 $\alpha$ could also segregate PC2 from SUMO, SUMO-bound Ubc9, and/or additional substrates. In either case, PC2HP1 $\alpha$ interaction would affect SUMO-PTM of multiple PC2 target proteins and regulate global levels of SUMOconjugation in the system.

SUMOylated HP1 $\alpha$ binds multiple euchromatin sites and exhibits a unique binding profile compared to unmodified HP1 $\alpha$. While HP1 $\alpha$ localizes to constitutive heterochromatin via association with established $\mathrm{H} 3 \mathrm{~K} 9 \mathrm{~m} 3$ marks, active chromatin sites lack H3K9m3. Hence it is unlikely SUMOylated HP1 $\alpha$ is recruited to most of the euchromatin via its $\mathrm{H} 3 \mathrm{~K} 9 \mathrm{~m} 3$ reader function. Alternatively, HP1 $\alpha$ binds $\alpha$-satellite ncRNA prior to $\mathrm{H} 3 \mathrm{~K} 9 \mathrm{~m} 3$ at pericentromeric heterochromatin in a SUMOdependent manner [14]. Similarly, SUMOylated HP1 $\alpha$ efficiently binds multiple chromatin-bound ncRNA including Rad51C ncRNA. Currently, it is unknown what drives this interaction between RNA and a SUMOmodified protein. HP1 $\alpha$ SUMOylation could initiate a conformational change in the linker domain to facilitate RNA binding. Additionally, a previous study identifies 7 critical residues of SUMO1 that allow for binding nucleic acids; 3 of these residues are conserved in SUMO3 [32]. SUMO3 could bind RNA in an analogous manner and therefore, covalent addition of SUMO3 to HP1 $\alpha$ could potentiate RNA binding. Extensive studies, beyond, the scope of this report, are required to clearly delineate this interaction.

HP1 $\alpha$ SUMOylation is dependent on SENP7L. We and others established that SENP7L (and HP1 $\alpha$ deSUMOylation) ensures the canonical function of $\mathrm{HP} 1 \alpha$ at $\mathrm{H} 3 \mathrm{~K} 9 \mathrm{~m} 3$-rich heterochromatin sites [5, 7, 24]. In contrast, SUMOylated HP1 $\alpha$ functions as a transcriptional repressor at multiple active promoter sites including DDR gene loci. Consistently, the reduced DNA repair defines DSB that persists in hyperSUMOylated $\mathrm{HP} 1 \alpha$ conditions in $\mathrm{BCa}$ cells. A recent report suggests HP1 $\alpha$ recruits SENP7L to the sites of DNA damage; interestingly, SENP7L mediates chromatin relaxation at
DNA damage foci independent of HP1 $\alpha$ deSUMOylation [6]. Collectively, the SENP7L-HP1 $\alpha$ interaction directs resolution of damaged DNA via 1) epigenetic regulated gene expression and/or 2) direct chromatin remodeling. Hence, it is not surprising that SENP7L and HP1 $\alpha$ SUMOylation define the efficacy of DNA-damaging chemotherapeutics in BCa cells. SENP7L levels predict the success rate of chemotherapeutics; specifically overall survival is increased over a 10 -year period.

Collectively our data demonstrates that the SUMO dynamics of the epigenetic remodeler HP1 $\alpha$ are tightly controlled in $\mathrm{BCa}$ cells and could predict chemotherapy efficacy as illustrated in Figure 7F. SUMO protease SENP7L is enriched at constitutive heterochromatin loci through its interaction with HP1 $\alpha$ and maintains hypoSUMOylated HP1 $\alpha$ levels in multiple cell types $[5-7,24]$. The SUMO E3 ligase PC2 also associates with the heterochromatic $\mathrm{H} 3 \mathrm{~K} 9 \mathrm{~m} 3$ mark (Figure $3 \mathrm{C}$ and 3D). In BCa cells with high SENP7L levels, HP1 $\alpha$ and PC2 are maintained in the unmodified state and readily interact. The interaction of PC2 with HP1 $\alpha$ reduces PC2's SUMO E3 ligase activity (Figure 1D) but supports PC2's localization at $\mathrm{H} 3 \mathrm{~K} 9 \mathrm{~m} 3$ sites (Figure $3 \mathrm{C}$ and 3D). Inversely, low SENP7L levels decrease PC2-HP1 $\alpha$ association (Figure 2C); this, in turn, enhances PC2's SUMO E3 ligase activity and SUMOylation of its target HP1 $\alpha$. SUMOylated HP1 $\alpha$ is more readily bound to active chromatin sites (Figure 4). The Rad51C gene locus is identified as a novel binding site for HP1 $\alpha$ (Figure 4C and 5A). SUMOylated HP1 $\alpha$ is enriched at the Rad51C gene and the recruitment of SUMOylated HP1 $\alpha$ to the Rad51C promoter requires chromatin-bound RNA (Figure 5A) specifically the chromatin-bound Rad51C ncRNA (Figure 5B). Recruitment of SUMOylated HP1 $\alpha$ to these loci substantially reduces the transcription of $\operatorname{Rad} 51 \mathrm{C}$ and other DNA-repair genes to potentiate DSB (Figure 5C and Figure 6). Consistently, BCa cells with elevated SUMOmodified HP1 $\alpha$ are more sensitive to DNA-damaging chemotherapeutics agents (Figure 7). Also patients on systemic chemotherapeutics with low SENP7L levels exhibit longer disease-free survival.

Although relatively efficacious, the majority of chemotherapeutic agents exhibit adverse toxicity. Hence, predicting chemotherapy sensitivity in $\mathrm{BCa}$ patients serves as an illusive holy grail in treatment. SENP7L and its regulation of HP1 $\alpha$ SUMOylation could be a good prognostic marker for chemotherapy responsiveness of $\mathrm{BCa}$ patients. Clearly more comprehensive studies with greater patient samples are required to validate this trend. 


\section{MATERIALS AND METHODS}

\section{Immunoprecipitation and western blotting of chromatin-bound proteins}

Isolation of chromatin-bound (CB) proteins was performed as defined previously [5]. The sample supernatant was incubated with antibodies for HP1 $\alpha$ and PC2 overnight and subsequently evaluated via SDSPAGE.

Cells were harvested, subject to SDS-PAGE, and immunoblotted as described previously $[5,33]$.

\section{In vitro SUMOylation}

In vitro SUMOylation was conducted using modifications to the protocol provided with the SUMOlink kit (Active Motif, Carlsbad, CA). Briefly, proteins were immunoprecipitated from cell lysates using the anti-Flag antibody bound to Protein A-agarose beads (Millipore, Billerica, MA). Immunoprecipitated proteins were incubated with the appropriate recombinant proteins and buffers as directed in the kit. After washes to reduce nonspecific interactions, proteins were resolved by SDSPAGE and analyzed by immunoblotting with antibodies against $\mathrm{HP} 1 \alpha$ and SUMO2/3.

\section{Chromatin immunoprecipitation (ChIP), ChIP- Seq, and RNase-ChIP}

The previously established protocols were used to conduct ChIP experiments [5]. Select ChIP samples were subject to Next Generation Sequencing. For this, the MDACC Sequencing and Microarray Core Facility prepared the DNA library construction and subsequently sequenced on the Illumina platform.

The sequencing reads were aligned to hg19 reference genome. Sequencing quality and calculate the mapping statistics was assessed with SAMSTAT. Partek Genomics Suite 6.6 was used to identify the binding regions. The detected peaks are filtered according to the $p$-values and the FDR set to 0.05 . The p-value was generated by comparing each ChIP sample to the Inputcontrol using a one-tailed binomial test.

For RNase-ChIP experiments, appropriately treated cells were incubated at room temperature for 5 minutes with $0.5 \%$ Triton X-100 (in PBS) to initiate permeabilization. Then permeabilized cells were treated with 5-Units RNase-H at room temperature for 5 minutes. Prior to initiating the ChIP process, the cells were fixed in $1 \%$ formaldehyde at room temperature for 10 minutes. Primers used real-time PCR to identify select gene promoter regions are provided with corresponding references (Supplemental Table S3, [5, 34, 35]).

\section{Chromatin RNA immunoprecipitation (RIP)}

Chromatin-RNA-Protein complexes were eluted and purified as directed in the RNA ChIP-it kit (Active Motif). Subsequently purified RNA was analyzed using real-time PCR and select primers for select ncRNA; list of primers and corresponding reference are included in Supplemental Table S4 [27, 36-39].

\section{Immunofluorescence, mitotic spread, and comet assay}

Immunofluorescence of whole cells and chromosome spreads were performed as outlined in previous publications [5]. The comet assay was performed according to manufacturer's instructions (Trevigen).

\section{Cell viability}

Cell viability was performed using the MTT assay as defined previously [40].

\section{Statistical analysis}

GraphPad Prism Version 4.0 (GraphPad Software) was used to conduct statistical analyses with either the Student's t-test or ANOVA employed to determine $p$ values of raw data. The Tukey's or Dunnett's post hoc test was performed to evaluate differences between groups or versus control with ANOVA, respectively. ChIP-Seq data was aligned by Burrows-Wheeler Aligner (BWA), a popularly used alignment tool [41]. The analysis was then performed using Partek software (Partek Inc).

\section{ACKNOWLEDGMENTS}

This work was supported in part by the Innovative Pilot Award to TBK from the Center for Cancer Epigenetics at UT MD Anderson Cancer Center.

\section{CONFLICTS OF INTEREST}

The authors declare no conflict of interest.

\section{Editorial note}

This paper has been accepted based in part on peerreview conducted by another journal and the authors' response and revisions as well as expedited peer-review in Oncotarget. 


\section{REFERENCES}

1. Bawa-Khalfe $\mathrm{T}$ and Yeh ET. SUMO Losing Balance: SUMO Proteases Disrupt SUMO Homeostasis to Facilitate Cancer Development and Progression. Genes Cancer. 2010; 1:748-752. doi: 10.1177/1947601910382555.

2. Cheng J, Bawa T, Lee P, Gong L and Yeh ET. Role of desumoylation in the development of prostate cancer. Neoplasia (New York, NY. 2006; 8:667-676.

3. Yeh ET, Gong L and Kamitani T. Ubiquitin-like proteins: new wines in new bottles. Gene. 2000; 248:1-14.

4. Bawa-Khalfe $\mathrm{T}$ and Yeh ET. The in vivo functions of desumoylating enzymes. Subcell Biochem. 2010; 54:170183.

5. Bawa-Khalfe T, Lu LS, Zuo Y, Huang C, Dere R, Lin FM and Yeh ET. Differential expression of SUMO-specific protease 7 variants regulates epithelial-mesenchymal transition. Proceedings of the National Academy of Sciences of the United States of America. 2012; 109:1746617471.

6. Garvin AJ, Densham RM, Blair-Reid SA, Pratt KM, Stone HR, Weekes D, Lawrence KJ and Morris JR. The deSUMOylase SENP7 promotes chromatin relaxation for homologous recombination DNA repair. EMBO reports. 2013; 14:975-983.

7. Maison C, Romeo K, Bailly D, Dubarry M, Quivy JP and Almouzni G. The SUMO protease SENP7 is a critical component to ensure HP1 enrichment at pericentric heterochromatin. Nat Struct Mol Biol. 2012; 19:458-460.

8. Bernstein E, Duncan EM, Masui O, Gil J, Heard E and Allis CD. Mouse polycomb proteins bind differentially to methylated histone $\mathrm{H} 3$ and RNA and are enriched in facultative heterochromatin. Molecular and cellular biology. 2006; 26:2560-2569.

9. Kang X, Qi Y, Zuo Y, Wang Q, Zou Y, Schwartz RJ, Cheng $\mathrm{J}$ and Yeh ET. SUMO-specific protease 2 is essential for suppression of polycomb group protein-mediated gene silencing during embryonic development. Molecular cell. 2010; 38:191-201.

10. Muchardt C, Guilleme M, Seeler JS, Trouche D, Dejean A and Yaniv M. Coordinated methyl and RNA binding is required for heterochromatin localization of mammalian HP1alpha. EMBO reports. 2002; 3:975-981.

11. Hayashi MT, Takahashi TS, Nakagawa T, Nakayama J and Masukata H. The heterochromatin protein Swi6/HP1 activates replication origins at the pericentromeric region and silent mating-type locus. Nat Cell Biol. 2009; 11:357362.

12. Panteleeva I, Boutillier S, See V, Spiller DG, Rouaux C, Almouzni G, Bailly D, Maison C, Lai HC, Loeffler JP and Boutillier AL. HP1alpha guides neuronal fate by timing E2F-targeted genes silencing during terminal differentiation. EMBO J. 2007; 26:3616-3628.
13. Ayyanathan K, Lechner MS, Bell P, Maul GG, Schultz DC, Yamada Y, Tanaka K, Torigoe K and Rauscher FJ, 3rd. Regulated recruitment of HP1 to a euchromatic gene induces mitotically heritable, epigenetic gene silencing: a mammalian cell culture model of gene variegation. Genes \& development. 2003; 17:1855-1869.

14. Maison C, Bailly D, Roche D, Montes de Oca R, Probst AV, Vassias I, Dingli F, Lombard B, Loew D, Quivy JP and Almouzni G. SUMOylation promotes de novo targeting of HP1alpha to pericentric heterochromatin. Nat Genet. 2011; 43:220-227.

15. Norwood LE, Moss TJ, Margaryan NV, Cook SL, Wright L, Seftor EA, Hendrix MJ, Kirschmann DA and Wallrath LL. A requirement for dimerization of HP1Hsalpha in suppression of breast cancer invasion. J Biol Chem. 2006; 281:18668-18676.

16. De Koning L, Savignoni A, Boumendil C, Rehman H, Asselain B, Sastre-Garau X and Almouzni G. Heterochromatin protein 1alpha: a hallmark of cell proliferation relevant to clinical oncology. EMBO molecular medicine. 2009; 1:178-191.

17. Kirschmann DA, Lininger RA, Gardner LM, Seftor EA, Odero VA, Ainsztein AM, Earnshaw WC, Wallrath LL and Hendrix MJ. Down-regulation of HP1Hsalpha expression is associated with the metastatic phenotype in breast cancer. Cancer research. 2000; 60:3359-3363.

18. Galanty Y, Belotserkovskaya R, Coates J, Polo S, Miller KM and Jackson SP. Mammalian SUMO E3-ligases PIAS1 and PIAS4 promote responses to DNA double-strand breaks. Nature. 2009; 462:935-939.

19. Wadman IA, Osada H, Grutz GG, Agulnick AD, Westphal $\mathrm{H}$, Forster A and Rabbitts TH. The LIM-only protein Lmo2 is a bridging molecule assembling an erythroid, DNAbinding complex which includes the TAL1, E47, GATA-1 and Ldb1/NLI proteins. EMBO J. 1997; 16:3145-3157.

20. Kirsh O, Seeler JS, Pichler A, Gast A, Muller S, Miska E, Mathieu M, Harel-Bellan A, Kouzarides T, Melchior $\mathrm{F}$ and Dejean A. The SUMO E3 ligase RanBP2 promotes modification of the HDAC4 deacetylase. EMBO J. 2002; 21:2682-2691.

21. Merrill JC, Melhuish TA, Kagey MH, Yang SH, Sharrocks $\mathrm{AD}$ and Wotton D. A role for non-covalent SUMO interaction motifs in Pc2/CBX4 E3 activity. PloS one. 2010; 5:e8794.

22. Yang SH and Sharrocks AD. The SUMO E3 ligase activity of Pc2 is coordinated through a SUMO interaction motif. Molecular and cellular biology. 2010; 30:2193-2205.

23. Stehmeier $\mathbf{P}$ and Muller S. Phospho-regulated SUMO interaction modules connect the SUMO system to CK2 signaling. Molecular cell. 2009; 33:400-409.

24. Romeo K, Louault Y, Cantaloube S, Loiodice I, Almouzni G and Quivy JP. The SENP7 SUMO-Protease Presents a Module of Two HP1 Interaction Motifs that Locks HP1 
Protein at Pericentric Heterochromatin. Cell reports. 2015.

25. Chu C, Qu K, Zhong FL, Artandi SE and Chang HY. Genomic maps of long noncoding RNA occupancy reveal principles of RNA-chromatin interactions. Molecular cell. 2011; 44:667-678.

26. Blume SW, Meng Z, Shrestha K, Snyder RC and Emanuel PD. The 5'-untranslated RNA of the human dhfr minor transcript alters transcription pre-initiation complex assembly at the major (core) promoter. Journal of cellular biochemistry. 2003; 88:165-180.

27. Martianov I, Ramadass A, Serra Barros A, Chow N and Akoulitchev A. Repression of the human dihydrofolate reductase gene by a non-coding interfering transcript. Nature. 2007; 445:666-670.

28. Tomikawa J, Shimokawa H, Uesaka M, Yamamoto N, Mori Y, Tsukamura H, Maeda K and Imamura T. Single-stranded noncoding RNAs mediate local epigenetic alterations at gene promoters in rat cell lines. J Biol Chem. 2011; 286:34788-34799.

29. Deng Z, Norseen J, Wiedmer A, Riethman H and Lieberman PM. TERRA RNA binding to TRF2 facilitates heterochromatin formation and ORC recruitment at telomeres. Molecular cell. 2009; 35:403-413.

30. Chen ES, Zhang K, Nicolas E, Cam HP, Zofall M and Grewal SI. Cell cycle control of centromeric repeat transcription and heterochromatin assembly. Nature. 2008; 451:734-737.

31. Somyajit K, Subramanya S and Nagaraju G. Distinct roles of FANCO/RAD51C protein in DNA damage signaling and repair: implications for Fanconi anemia and breast cancer susceptibility. J Biol Chem. 2012; 287:3366-3380.

32. Eilebrecht S, Smet-Nocca C, Wieruszeski JM and Benecke A. SUMO-1 possesses DNA binding activity. BMC research notes. 2010; 3:146.

33. Bawa-Khalfe T, Cheng J, Wang $Z$ and Yeh ET. Induction of the SUMO-specific protease 1 transcription by the androgen receptor in prostate cancer cells. J Biol Chem. 2007; 282:37341-37349.
34. Cawthon RM. Telomere measurement by quantitative PCR. Nucleic acids research. 2002; 30:e47.

35. Rabinovich A, Jin VX, Rabinovich R, Xu X and Farnham PJ. E2F in vivo binding specificity: comparison of consensus versus nonconsensus binding sites. Genome research. 2008; 18:1763-1777.

36. Kaneko S, Li G, Son J, Xu CF, Margueron R, Neubert TA and Reinberg D. Phosphorylation of the PRC2 component Ezh2 is cell cycle-regulated and up-regulates its binding to ncRNA. Genes \& development. 2010; 24:2615-2620.

37. Ganesan S, Silver DP, Greenberg RA, Avni D, Drapkin R, Miron A, Mok SC, Randrianarison V, Brodie S, Salstrom J, Rasmussen TP, Klimke A, Marrese C, Marahrens Y, Deng CX, Feunteun J, et al. BRCA1 supports XIST RNA concentration on the inactive X chromosome. Cell. 2002; 111:393-405.

38. Yang L, Lin C, Liu W, Zhang J, Ohgi KA, Grinstein JD, Dorrestein PC and Rosenfeld MG. ncRNA- and Pc2 methylation-dependent gene relocation between nuclear structures mediates gene activation programs. Cell. 2011; 147:773-788.

39. Sampl S, Pramhas S, Stern C, Preusser M, Marosi C and Holzmann K. Expression of telomeres in astrocytoma WHO grade 2 to 4: TERRA level correlates with telomere length, telomerase activity, and advanced clinical grade. Translational oncology. 2012; 5:56-65.

40. Kumar S, Prasad S and Sitasawad SL. Multiple antioxidants improve cardiac complications and inhibit cardiac cell death in streptozotocin-induced diabetic rats. PloS one. 2013; 8:e67009.

41. Li H and Durbin R. Fast and accurate short read alignment with Burrows-Wheeler transform. Bioinformatics. 2009; $25: 1754-1760$ 